\title{
Sur la séparation des pertes dans une turbine Francis
}

\section{Differentiation of losses in Francis turbines}

\author{
PAR F. FONTAINE GT P. CAMPMAS
}

\begin{abstract}
Le contrôle de rendement global d'une turbine Francis permet à l'exploitant d'utiliser des groupes neufs dans des conditions optima. Il est non moins intéressant de suivre l'évolution de ce rendement dans le temps, notamment en fonction de l'usure des différents organes et de permettre ainsi la justification du remplacement ou de la réparation des pièces usées.

Une étude antérieure, publiée dans la Houlle Blanche de janvier et février 1951 sous le titre « Quelques applications de la méthode thermométrique Poirson $»$, donnait un mode de mesure des pertes dans les labyrinthes d'une turbine Francis.

La présente étude complète la précédente et donne un moyen de mesurer les pertes dans l'aspiration et dans l'ensemble : robinets sphériques, bâche, distributeur et roue.

II apparaît, ainsi, qu'il est possible de « séparer 》 les pertes d'une turbine Francis comme on sépare les pertes d'un générateur électrique quelconque.
\end{abstract}

\begin{abstract}
A check-up of the total efficiency of a Francis Turbine malies possible the use of new units under optimum conditions. If is of no less interest to follow the evolution of the efficiency over a period of time, especially as a function of the wear of the various parts, it thereby becoming possible to justify the replacing or the repairing of worn parts.

Former investigations (no. 1/1951" some applications of Poirson's Thermometrical method") gave a melhod for measuring losses in Francis Turbine labyrinths. The present study completes these investigations and provides a means of measuring the losses in the draft tube and in the spherical balve-casing-distributor-runner unit.

It would thus seem that it is possible to separate Francis turbine losses in the same waly as the losses of any electric generator.
\end{abstract}

\section{I. - GENERALITÉS}

Au cours de travaux antérieurs, nous avons recherché l'importance des pertes aux labyrinthes, l'influence des divers facteurs qui interviennent et les moyens de réduire ces pertes. Mais cette étude n'est qu'un des aspects d'un problème beaucoup plus général que nous nous proposons d'aborder aujourd'hui : la séparation des pertes dans une turbine Francis.

Il est inutile de souligner l'intérêt de cette séparation qui, nécessitant une étude plus détaillée du bilan énergétique des turbines, peut orienter avec profit les recherches sur l'augmentation du rendement global.

Nous inspirant du trajet de l'eau schématisé ci-dessous, nous distinguerons :

- Les pertes par frottement, turbulence, dans la vanne, la bâche, le distributeur et la roue proprement dite;

- Les pertes dans les labyrinthes;

- Les pertes dans l'aspiration. 


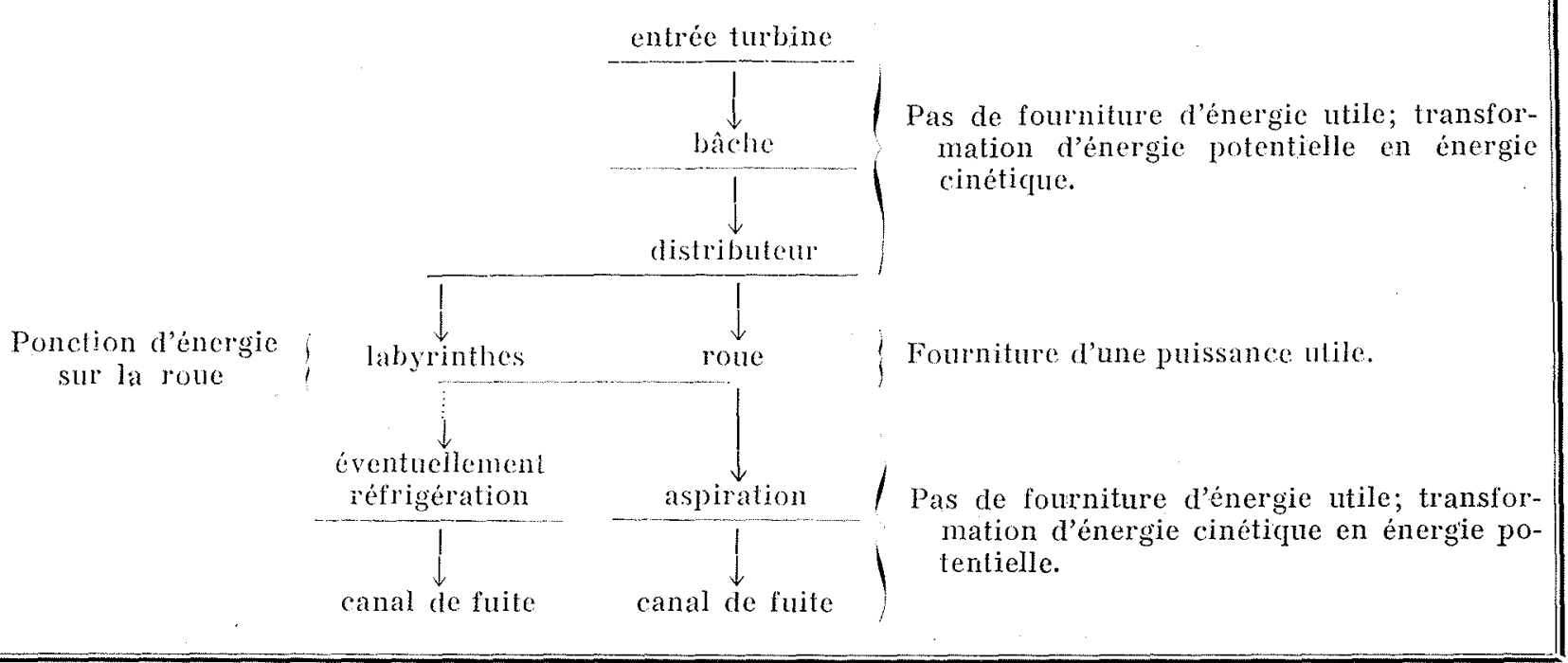

Enfin, pour mémoire, rappelons que le rendement global de la turbine tient compte des per. tes par frottement des paliers sur l'arbre.

Pour des commodités d'exposé, nous étudie- rons d'abord les pertes dans les labyrinthes et l'aspiration, rejetant l'ctude de la roue dans la dernière partie.

\section{II. - PERTES AUX LABYRINTHES}

Nous avons déjà abordé cette question dans un compte rendu antérieur (la Houille Blanche, janvier-février 1951 : «Quelques applications de la méthode thermométrique Porrson. $\gg$ ).

Rappelons très brievement que l'on peut distinguer :

$1^{\circ}$ Les pertes d'énergie correspondant au débit de fuite $q$ des labyrinthes sous la chute $\mathrm{H}_{n}$, soit $q \mathrm{H}_{n}$. Ces pertes dépendent :

- Des dimensions géométriques des labyrinthes. En particulier, les fuites augmentent avec le jeu des labyrinthes.

- De l'état des surfaces en regard.

- De la pression statique à l'entrée des labyrinthes. Ce dernier paramètre varie avec la charge de la turbine : si, pour une hauteur de chute nette donnée, on augmente. la charge d'une turbine Francrs, on constate que la vitesse de l'eau dans le distributeur diminue, ce qui entraîne une augmentation de la pression statique à l'entrée des labyrinthes, done une augmentation du débit de fuite.

$2^{\circ}$ Les pertes par « frottements fluides 》 des filets liquides les uns contre les autres. Elles constitueraient sensiblement les pertes totales aux labyrinthes si le débit de fuite était nul et se traduisent par une ponction d'énergie sur la roue. Elles sont fonctions :

- Des dimensions géométriques. En particulier, ces pertes augmentent quand le jeu des labyrinthes diminue.

- De l'état des surfaces en regard. Le poli diminue ces pertes (mais augmente probablement le débit de fuite).

- De la puissance quatrieme environ du diamètre des labyrinthes.

- Enfin, pour mémoire, de la vitesse de rotation des turbines.

Remarquons bien que certains paramètres (jeu, poli des surfaces) ne permettent de diminuer une catégorie de pertes qu'en augmentant parallèlement la deuxième catégorie. Ce phénomène est illustré par l'exemple numérique donné ci-après : tableaux I et II.

Par contre, la diminution du diamètre $D$ des labyrinthes entraine la diminution, dans des proportions importantes, des deux catégories de pertes :

-- Les pertes par «frottements fluides» diminuent rapidement.

- En appelant $\varepsilon$ le jeu aux labyrinthes et en admettant qu'on prenne $\varepsilon=a \mathrm{D}(a$ : cons- 
tante), la section de passage de l'eau varie comme $\varepsilon \mathrm{D}=a \mathrm{D}^{2}$ et, par suite, le débil de fuite $q$ diminue comme $\mathrm{D}^{2}$ (toutes choses restant égales par ailleurs).
D'où l'intérêt de rapprocher au maximum les labyrinthes de l'axe de la turbine (modification actuellement réalisée par certains constructeurs, dans la mesure où elle est possible).

\section{Valeurs numériques des pertes à Brommat ${ }^{1}$}

\section{1. --.- MÉthode DE MEsuRe}

A Brommat, l'eau de fuite du labyrinthe supérieur peut indifféremment être rejetée dans l'aspirateur ou refoulée dans un bassin, sous la voûte de l'usine, d'où elle alimente les divers circuits de réfrigération. Par suite, la mesure du débit de fuite $q_{s}$ du labyrinthe supérieur peut se ramener à la mesure du débit dans la conduite de refoulement. Des considérations d'ordre matériel nous ont conduits à utiliser un tube de Príot.

Les labyrinthes supérieur et inférieur étant identiques, le débit de fuite $q_{i}$ du labyrinthe inférieur a été admis égal à :

$$
q_{i}=q_{s} \sqrt{\frac{\mathrm{P}_{0}-\mathrm{P}_{1}}{\mathrm{P}_{0}-\mathrm{P}_{2}}}
$$

avec :

$\mathrm{P}_{0}$ pression à l'entrée des labyrinthes;

$P_{1}$ pression dans l'aspirateur à la sortie de la roue;

$\mathrm{P}_{1}$ pression de refoulement à la sortie du labyrinthe supérieur.

Enfin, la mesure des pertes totales a été faite en utilisant la méthode thermométrique.

\section{$2^{\circ}$ Résultats}

A titre indicatif, nous donnons la valeur des débits de fuite et des pertes d'énergie pour deux groupes ne différant entre eux que par le jeu laissé aux labyrinthes.

On en déduit les courbes de la figure 1.

\section{Remaroues :}

$1^{\circ}$ Comme l'eau du labyrinthe supérieur est utilisée en contre-pression pour alimenter les circuits de réfrigération, on peut dire que

1. Caractéristiques des groupes : hauteur de chute: 240 à $260 \mathrm{~m}$; vitesse : $500 \mathrm{trs} / \mathrm{mn}$; puissance : $32.500 \mathrm{kVA}$,
Tableau 1

GROUPE 4. - JEU $9 / 10 \mathrm{~mm}$

\begin{tabular}{|c|c|c|c|c|c|c|}
\hline \multirow[b]{2}{*}{$\begin{array}{l}\text { Puissance } \\
\text { aux bornes } \\
\text { de } \\
\text { l'alternateur } \\
\text { (kW) }\end{array}$} & \multirow[b]{2}{*}{$\begin{array}{l}q_{s} \\
1 / \mathrm{s}\end{array}$} & \multirow[b]{2}{*}{$\begin{array}{l}q_{i} \\
1 / \mathrm{s}\end{array}$} & \multirow[b]{2}{*}{ 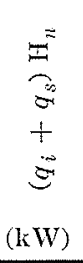 } & \multirow[b]{2}{*}{ 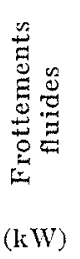 } & \multicolumn{2}{|c|}{ Pertes totales } \\
\hline & & & & & $(\mathrm{kW})$ & 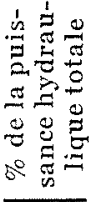 \\
\hline $0 \ldots$ & 48 & 53 & 250 & & 950 & 32 \\
\hline $5.000 \ldots \ldots$ & 100 & 110 & 525 & & 1.225 & 15 \\
\hline $10.000 \ldots \ldots$ & 122 & 134 & 640 & & 1.340 & 9,8 \\
\hline $15.000 \ldots \ldots$ & 127 & 140 & 665 & 700 & 1.365 & 7,3 \\
\hline $20.000 \ldots$ & 145 & 160 & 760 & & 1.460 & 6,1 \\
\hline $25.000 \ldots$ & 168 & 185 & 880 & & 1.580 & 5,4 \\
\hline $30.000 \ldots \ldots$ & 180 & 198 & 945 & & 1.645 & 4,7 \\
\hline $32.000 \ldots$ & 185 & 203 & 960 & & 1.660 & 4,3 \\
\hline
\end{tabular}

Tableau II

GROUPE 6. - JEU $14.10 \mathrm{~mm}$

\begin{tabular}{|c|c|c|c|c|c|c|}
\hline \multirow[b]{2}{*}{$\begin{array}{l}\text { Puissance } \\
\text { aux bornes } \\
\text { de } \\
\text { l'alternateur } \\
\text { (kW) }\end{array}$} & \multirow[b]{2}{*}{$\begin{array}{c}q_{s} \\
1 / s\end{array}$} & \multirow[b]{2}{*}{$\begin{array}{l}q_{i} \\
1 / \mathrm{s}\end{array}$} & \multirow[b]{2}{*}{$\begin{array}{l}\overrightarrow{x^{2}} \\
\approx \\
+ \\
\tilde{\Xi} \\
(\mathrm{kW})\end{array}$} & \multirow[b]{2}{*}{ 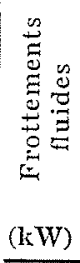 } & \multicolumn{2}{|c|}{ Pertes totales } \\
\hline & & & & & $(\mathrm{kW})$ & 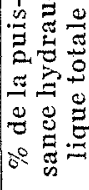 \\
\hline 0. & 144 & 158 & 755 & & 1.325 & 44 \\
\hline $5.000 \ldots \ldots$ & 192 & 211 & 1.010 & & 1.580 & 19 \\
\hline $10.000 \ldots \ldots$ & 216 & 237 & 1.130 & 570 & 1.700 & 12,4 \\
\hline $15.000 \ldots \ldots$ & 228 & 251 & 1.200 & & 1.770 & 9,5 \\
\hline $20.000 \ldots$ & 240 & 264 & 1.255 & & 1.825 & 7,5 \\
\hline 25.000 . & 255 & 280 & 1.335 & & 1.905 & 6,6 \\
\hline 30.000 & 290 & 320 & 1.520 & & 2.090 & 6 \\
\hline
\end{tabular}




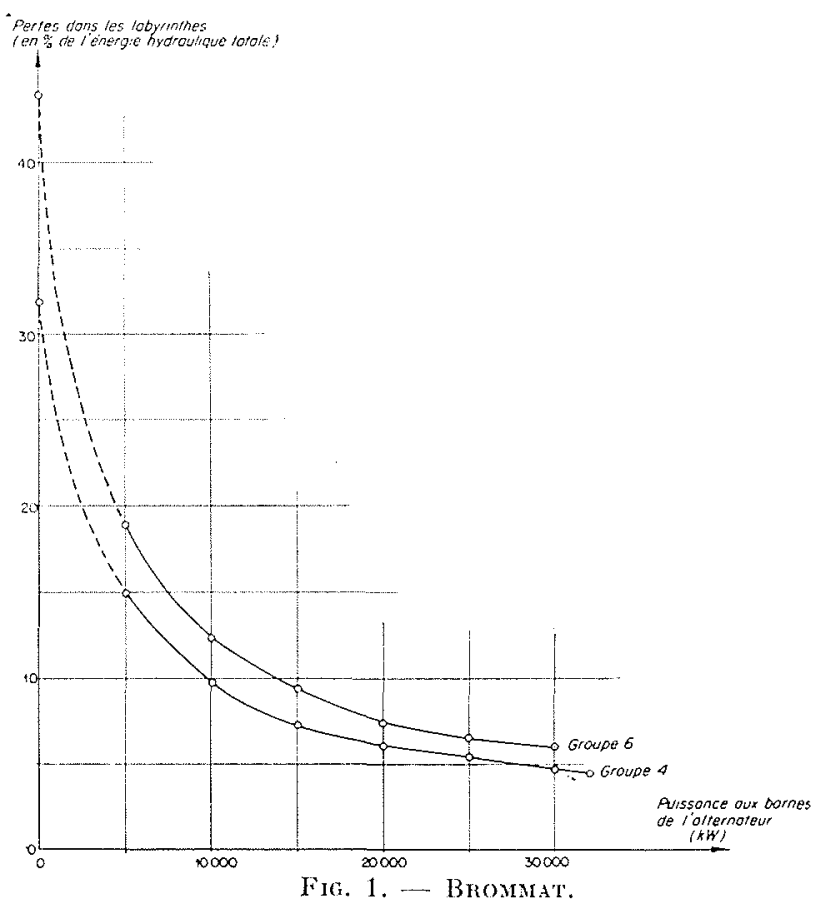

Puissance perduc dans les labyrinthes. les pertes déncrgie imputées au labyrinthe doirent être en réalité imputces a l'ensemble :

labyrinthe - circuit de refrigération.

¿ Si l'on compare les pertes par frottements lluides des groupes 5 et 6 (jeux egaux, mais lormes différentes), on trouve :

Groupe $6: 570 \mathrm{~kW}$

Groupe $5: 590 \mathrm{~kW}$

Or l'application des formules de calcul des pertes donne un rapport de 1,4 , soit :

$$
\frac{\text { pertes sroupe } 6}{\text { pertes groupe } 5}=1,4
$$

La différence avec le rapport $(0,97)$ mesuré expérimentalement doit probablement être attribuée au poli des surfaces réalisé sur le groupe 6 .

Nous venons d'ailleurs de pousser encore davantage le poli des surfaces sur une autre turbine et nous attendons les résultats d'un nouvel essai.

\section{III. - PERTES DANS L'ASPIRATION 1}

\section{Position du probleme}

A la sortie de la roue, et compte tenu de ce que l'aspirateur a joué son rôle de transformateur, l'eau possède une certaine énergie totale $\mathrm{T}_{1}$. Précisons que ' $\mathrm{T}_{1}$ désigne l'énergie que l'on peut recueillir en ramenant cette eau :

- A la cote de restitution de la turbine;

- A la pression atmosphérique;

- A une vitesse nulle

(la transformation se faisant à température constante et sans modification physique ou chimique).

Remarquons que l'aspirateur ne fournit à l'extérieur aucune énergie utile. 'T, représente donc les pertes dans l'aspiration.

Le principe de la conservation de l'énergie nous permet d'écrire :

$$
\mathrm{T}_{1}=\mathrm{T}_{p}+\mathrm{T}_{2}
$$

$\mathrm{T}_{p}$ étant l'énergie dissipée par frottements, turbulence, etc., dans l'aspiration. $\mathrm{T}_{p}$ se traduit par échauffement de l'eau.

1. Nous inclurons l'énergie résiduelle de l'eau à la sortie du tuyau d'aspiration dans cette catégorie de pertes, bien que cette énergie soit en partic récupérée dans le canal de fuite et se traduise par un abaissement du plan d'eau aval au débouché de l'aspirateur.
' $\mathrm{T}_{2}$ étant l'énergie résiduelle de l'eau à la sortic de l'aspiration.

\section{2" Evaluation des pertes dans l'aspiration}

L'expérience montre que, en un point donné M de l'aspiration, la vitesse et la pression ne sont pas constantes dans le temps mais oscillent autour de valeurs moyennes que nous désignerons par $v$ et $p$ (régime pulsatoire). L'équation de Bernoular n'est donc pas applicable. Nous admettrons cependant en première approximation que l'énergie d'une masse d'eau $d m$ située au point $M$ est :

$$
d \mathrm{~T}=g d m\left(\frac{v^{2}}{2 g}+\frac{p}{\pi}+z\right)
$$

$p$ : pression relative, positive ou négative.

$z$ élant la cote du point M.

Soit (פ) une section du còne d'aspiration au voisinage de la roue, $M$ un point de ( $\mathbf{\Sigma}), \approx$ l'angle de la vitesse de l'eau et de la normale à $(\Sigma)$ en ce point. Enfin, nous appellerons $d w$ la puissance que peut fournir un débit $d Q$ traversant un élément de surface $d \sigma$ de $(\Sigma)$ entourant le point $M$ quand on ramène l'eau aux conditions limites : 
cote de restitution, pression atmosphérique, vitesse nulle, sans modifier sa température ni son état physique ou chimique.

$$
\begin{aligned}
& \text { On a : } \quad d w=\bar{\omega} d Q\left(\frac{v^{2}}{2 g}+\frac{p}{\omega}+z\right) \\
& \text { Or : } \quad d Q=v \cos \alpha d \sigma \\
& \text { Done : } \\
& d w=\Phi\left(\frac{v^{3} \cos \alpha}{2 g}+\frac{p \nu \cos \alpha}{\sigma}+z v \cos \alpha\right) d \sigma
\end{aligned}
$$

On en déduit la puissance perdue dans l'aspiration :

$$
w_{1}=\bar{\sigma}\left(\begin{array}{c}
1 \\
2 g
\end{array} \iint_{(\Sigma)} v^{*} \cos \alpha d \sigma+\frac{1}{\sigma} \iint_{(\Sigma)} p v \cos \alpha d \sigma+\iint_{(\Sigma)} z v \cos \alpha d \sigma\right)
$$

Définissons trois grandenirs $v_{1}, p_{1}$ et $z_{1}$ par les relations :

$$
\left.\begin{array}{rl}
Q_{1} v_{1}{ }^{2} & =\iint_{(\Sigma)} p^{\prime \prime} \cos \alpha d \sigma \\
Q_{1} p_{1} & =\iint_{(\Sigma)} p v \cos \alpha d \sigma \\
Q_{1} z_{1} & =\iint_{(\Sigma)} z v \cos \alpha d \sigma
\end{array}\right\}
$$

étant le débit traversant la surface $(\Sigma)$.

$$
w_{1}=\bar{\omega} \mathrm{Q}_{1}\left(\frac{v_{1}^{2}}{2 g}+\frac{p_{1}}{i j}+z_{1}\right)
$$

Soit $Q$ le débit absorbé par la turbine, $H_{n}$ la hauteur de chute nette.

La puissance hydraulique disponible est :

$$
w=-\varpi \mathrm{Q} \mathrm{H}_{u}
$$

Posolns :

$$
h=\frac{v_{1}^{2}}{2 g}+\frac{p_{1}}{\pi}+z_{1}
$$

et confondons $Q$ et $Q_{1}$ (la différence $Q-Q_{1}$, suivant les cas nulle ou égale au débit traversant le labyrinthe supérieur, étant généralement négligeable devant $Q$, sauf peut-être pour les marches à vide de certaines turbines).

On peut alors écrire :

$$
\frac{w_{1}}{w !}=\frac{h}{\mathrm{H}_{n}}
$$

Les pertes dans l'aspiration, exprimées en \% de l'énergie hyraulique totale, sont égales à $100 \frac{h}{\mathrm{H}_{n}}$.

Remarque. - On a supposé implicitement que le poids spécifique de l'eau reste constant, donc qu'à la sortie de la roue, l'eau ne se présente pas sous forme d'émulsion. Cette hypothèse semble vérifiée par les soutirages que nous avons effectués en divers points du cône d'aspiration.

3" Disposite expérimental Utilisté a Bromat

a) Simplification dans le cas particulier des turbines verticales:

La première partic de l'aspirateur étant constituée par un cône de révolution vertical placé sous la roue, on peut prendre pour $(\Sigma)$ une section droite horizontale de cote $z_{1}$ et admettre que la répartition des vitesses (en grandeur et direction) et celle des pressions le long d'un rayon de la section sont indépendantes du rayon choisi.

En désignant par $r$ la distance du point $M$ au centre de la section et par $R$ le rayon de la section, les relations (1) s'écrivent :

$$
\begin{aligned}
\mathrm{Q}_{1} v_{1}^{2} & =2 \pi \int_{0}^{\mathrm{R}} v^{3} \cos \alpha r d r \\
\mathrm{Q}_{1} p_{1} & =2 \pi \int_{0}^{\mathrm{R}} p v \cos \alpha r d r \\
z_{1} & =z_{1}
\end{aligned}
$$

On est donc ramené à mesurer la vitesse (en grandeur et direction) et la pression en divers points d'un rayon arbitraitement choisi.

\section{b) Appareils utilisés:}

Les mesures ont été faites avec un tube de PrTot dont nous reproduisons une coupe (fis. 2); grâce à un guide boulonné sur le cône d'aspiration et muni d'un presse-étoupe, la partie active du tube peut occuper toutes les positions perpendiculaires à un rayon de la section étudiée.

Pour la mesure des pressions, nous avons utilisé 2 manometres métalliques, l'un gradué de $-76 \mathrm{~cm} \mathrm{Hg}$ à $1 \mathrm{~kg} / \mathrm{cm}^{2}$, l'autre de 0 à $5 \mathrm{~kg} / \mathrm{cm}^{2}$; les manomètres étaient fixés sur un pot d'amortissement.

Les angles du tube de PrTot avec la verticale. étaient lus sur un simple rapporteur. La direc- 
tion de la vitesse en un point élait déterminéc en prenant la moyenne (algébrique) de deux angles correspondant à une même pression dynamique nettement inférieure à la pression dyna-

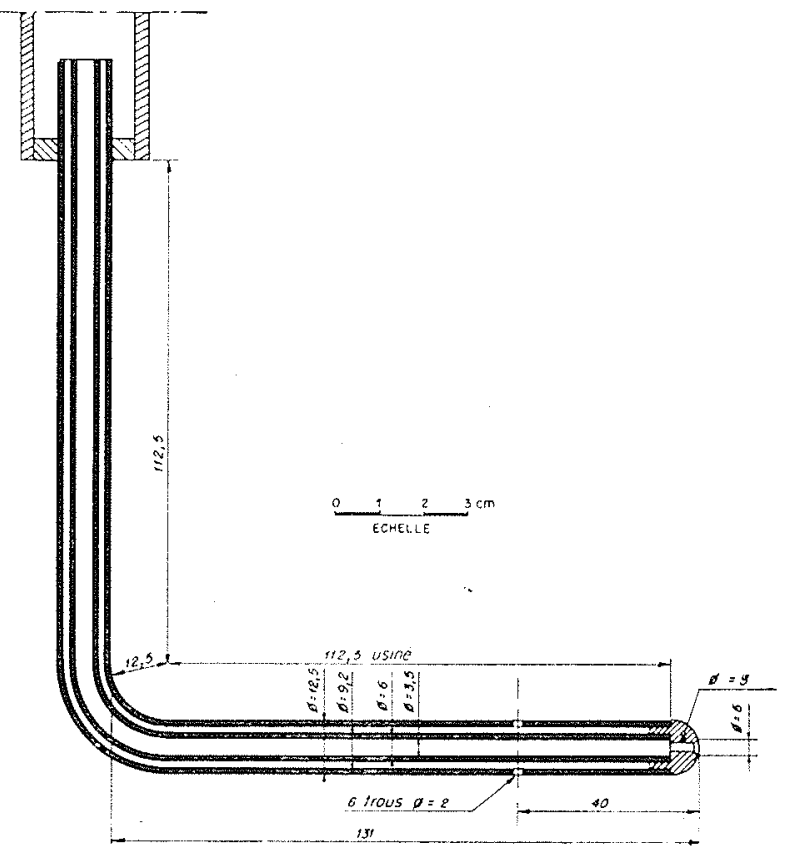

FIG. 2 .

Coupe du tube de Pitot.

mique maximum; en effet si, pour un point donné de l'aspirateur, on trace la courbe représentant la pression dynamique en fonction de l'angle du tube de PITot avec la verticale, on obtient une courbe (en cloche) présentant un

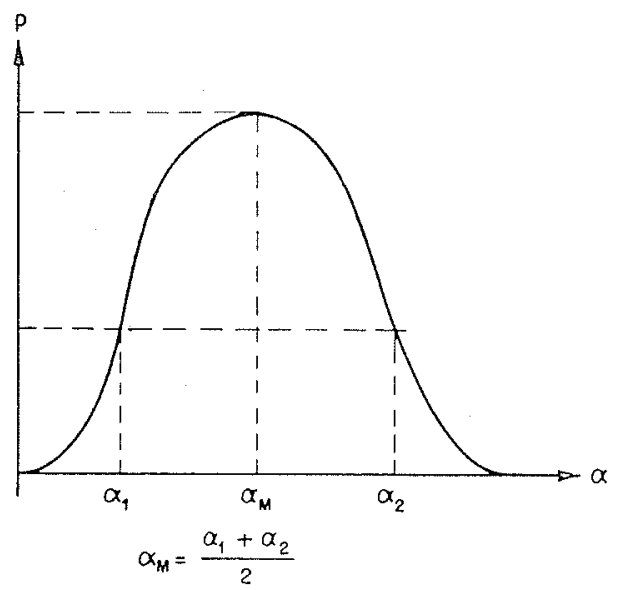

maximum assez aplati (avec nos moyens de mesure), d'où une grande imprécision dans la recherche directe de l'angle correspondant au maximum.

\section{Remaroues :}

$1^{\circ}$ Dans ces mesures, nous ne tenons pas compte de la composante radiale de la vitesse, bien que cette composante ne soit pas nulle : nous avons mis son existence en évidence avec des tubes de PrTot dont la partie active faisait des angles avec le rayon de mesure s'échelonnant entre $30^{\circ}$ fermé et $30^{\circ}$ ouvert (en passant évidemment par $90^{\circ}$ ).

$2^{\circ}$ Des difficultés matérielles nous ont interdit des mesures immédiatement à la sortie de la roue. La section étudiée est à $0,50 \mathrm{~m}$ environ au-dessous de la roue.

\section{$4^{\circ}$ Résultats observés a Brommat}

Les conditions des mesures étaient :

Rayon de la section de mesure, $660 \mathrm{~mm}$.

Position de cette section, $0,50 \mathrm{~m}$ au-dessous de la roue.

Les angles sont comptés positivement quand l'eau tourne en sens inverse de la rone de la turbine.

p désigne la pression relative mesurée, affectéc de son signe.

Les résultats des essais sont consignés dans les tableaux ci-dessous.

I

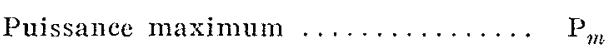

Chute nette ............... $\mathrm{H}_{n}=243 \mathrm{~m}$

Plan d'eau aval (au-dessous de la sec-

tion de mesure) $\dot{a} \ldots \ldots \ldots \ldots \ldots \ldots 1 \mathrm{~m}$

\begin{tabular}{|c|c|c|c|}
\hline $\begin{array}{c}r \\
\text { (en mm) }\end{array}$ & $\begin{array}{c}p / \overline{6} \\
\text { en m d'eau) }\end{array}$ & $\begin{array}{c}v \\
\text { (en m/s) }\end{array}$ & $\begin{array}{c}q \\
\text { (en degrés) }\end{array}$ \\
\hline 633 & -3 & 8,3 & -5 \\
575 & $-2,45$ & 13,95 & $\cdots 1$ \\
515 & -3 & 15,65 & -20 \\
465 & $-4,07$ & 16,4 & $-17,5$ \\
405 & $-4,07$ & 17,5 & $-12,5$ \\
350 & $-5,45$ & 18,25 & -15 \\
275 & $-7,5$ & 18,25 & -15 \\
205 & $-7,1$ & 0 & \\
130 & $-7,1$ & 0 & \\
\hline
\end{tabular}


Puissance $\ldots \ldots \ldots \ldots \ldots \ldots \ldots \ldots \ldots$. $3 / 4 \mathrm{P}_{m}$

Chute nette .............. $\mathrm{H}_{n}=248 \mathrm{~m}$

Plan d'eau aval (au-dessous de la sec-

tion de mesure) à . . . . . . . 1,20 m

\begin{tabular}{|c|c|c|c|}
\hline $\begin{array}{c}r \\
(\mathrm{en} \mathrm{mm})\end{array}$ & $\begin{array}{c}p / \bar{\omega} \\
(\mathrm{en} \mathrm{m} \text { d'eau })\end{array}$ & $\begin{array}{c}v \\
(\mathrm{en} \mathrm{m} / \mathrm{s})\end{array}$ & $\begin{array}{c}\alpha \\
\text { (en degrés) }\end{array}$ \\
\hline 605 & $-4,35$ & 9,2 & $-37,5$ \\
560 & $-4,75$ & 12,2 & -10 \\
505 & $-4,35$ & 12 & 0 \\
435 & $-4,35$ & 11,6 & 0 \\
385 & $-4,35$ & 12 & 2,5 \\
320 & $-4,18$ & 12,5 & 0 \\
260 & $-4,18$ & 12,65 & 4 \\
205 & $-4,75$ & 14 & 2,5 \\
130 & $-4,35$ & 13,9 & 0 \\
\hline
\end{tabular}

III

Puissance $\ldots \ldots \ldots \ldots \ldots \ldots \ldots \ldots{ }_{2 / 3} \mathrm{P}_{m}$

Chute nette $\ldots \ldots \ldots \ldots \ldots \ldots \ldots \ldots \mathrm{H}_{n}=247 \mathrm{~m}$

Plan deau aval (au-dessous de la sec-

tion de mesure) à $\ldots \ldots \ldots \ldots \ldots, 1,20 \mathrm{~m}$

\begin{tabular}{|c|c|c|c|}
\hline $\begin{array}{c}r \\
(\mathrm{en} \mathrm{mm})\end{array}$ & $\begin{array}{ll}p / \pi \\
(e n \mathrm{~m} d \text { 'eau }\end{array}$ & $\left(e n \begin{array}{c}v \\
(\mathrm{~m} / \mathrm{s})\end{array}\right.$ & $\frac{\alpha}{(\text { cul degles }}$ \\
\hline 625 & $-\cdots 2,7$ & 10,55 & -30 \\
\hline 565 & $-\cdots-3$ & 10,2 & $\cdots-20$ \\
\hline 505 & $-\ldots-3$ & 10,2 & $-\cdots 12,5$ \\
\hline 485 & $-\ldots-3$ & 11.1 & -15 \\
\hline 385 & $-3,4$ & 12,45 & --17.5 \\
\hline 336 & $-3,4$ & 12,8 & $-22,5$ \\
\hline 285 & $-\quad 4,35$ & 12,4 & $\ldots 27,5$ \\
\hline 260 & $\ldots \ldots .4,35$ & 5,7 & $-\ldots-30$ \\
\hline 215 & $\cdots \cdots \cdot 4,3$ & () & \\
\hline 130 & $\cdots-4,35$ & 0 & \\
\hline
\end{tabular}

Puissance $\ldots \ldots \ldots \ldots \ldots \ldots \ldots \ldots 1 / 3 \mathrm{P}_{m}$

Chute nette $\ldots \ldots \ldots \ldots \ldots \ldots \ldots \ldots \mathrm{H}_{n}=249 \mathrm{~m}$

Plan d'eau aval (au-dessous de la section de mesure) à ........... $1 \mathrm{~m}$

\begin{tabular}{|c|c|c|c|}
\hline $\begin{array}{c}r^{\prime} \\
\text { (en mm) }\end{array}$ & $\begin{array}{c}p / \bar{\omega} \\
\text { (en } \mathrm{m} \mathrm{d'eau)}\end{array}$ & $\begin{array}{c}v \\
(\mathrm{en} \mathrm{m} / \mathrm{s})\end{array}$ & $\begin{array}{c}\alpha \\
\text { (en degrés) }\end{array}$ \\
\hline 615 & 0 & 17,4 & -50 \\
570 & $-1,35$ & 15,8 & -45 \\
535 & $-2,85$ & 15,6 & $-42,5$ \\
505 & $-2,7$ & 9,3 & $-47,5$ \\
485 & $-3,1$ & 7,8 & $-47,5$ \\
450 & $-3,1$ & 0 & \\
130 & $-3,1$ & 0 & \\
\hline
\end{tabular}

Remarquons immédiatement que la précision des résultats numériques ne correspond pas à la précision réelle des mesures.

Par intégrations graphiques, on en déduit $h$ et par suite la courbe donnant les pertes dans laspiration (en fonction de la charge du groupe) figure 3.

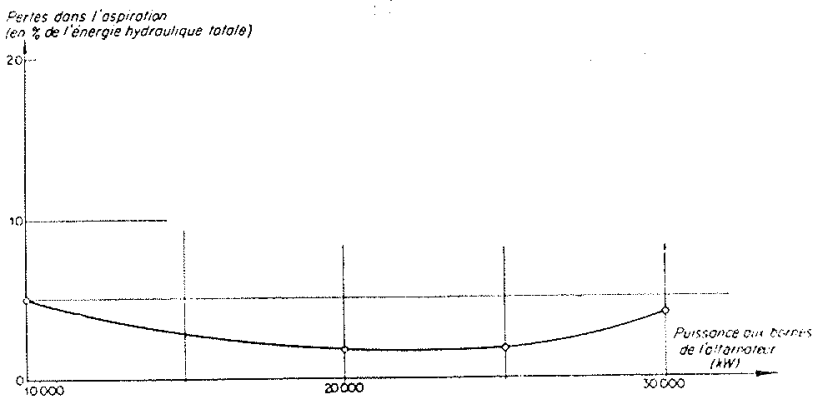

Fig. 3. - Brommat (groupe 4).

Puissance perdue dans l'aspiration.

Nous reproduisons, à titre indicatif, les cour" bes représentant :

$$
\frac{v^{\ddot{c} \cos \alpha}}{2 g} \text { et } \frac{p v}{\pi} \cos \alpha
$$

en fonction de $r^{2}$ pour diverses charges afin de montrer diverses « répartitions d'énergie » que nous avons rencontrées (fig. 4).

Remarquons que vraisemblablenent dans les zones considérées comme plages de vitesse nulle, l'eau a des vitesses qui peuvent être non négligeables tout en n'étant pas décelables avec nos 
moyens de mesure. En particulier, il est probable que la zone centrale du tuyau d'aspiration est, à pleine charge, le siège de mouvements tourbillonnaires, et il n'est pas exclu qu'il existe des vitesses ascendantes.

Enfin, précisons que nous ne prétendons pas que les pertes d'énergic localisćes dans l'aspiration lui soient en totalité imputables. Il est bien évident qu'il y a interaction plus ou moins étroite entre la roue et l'aspirateur.

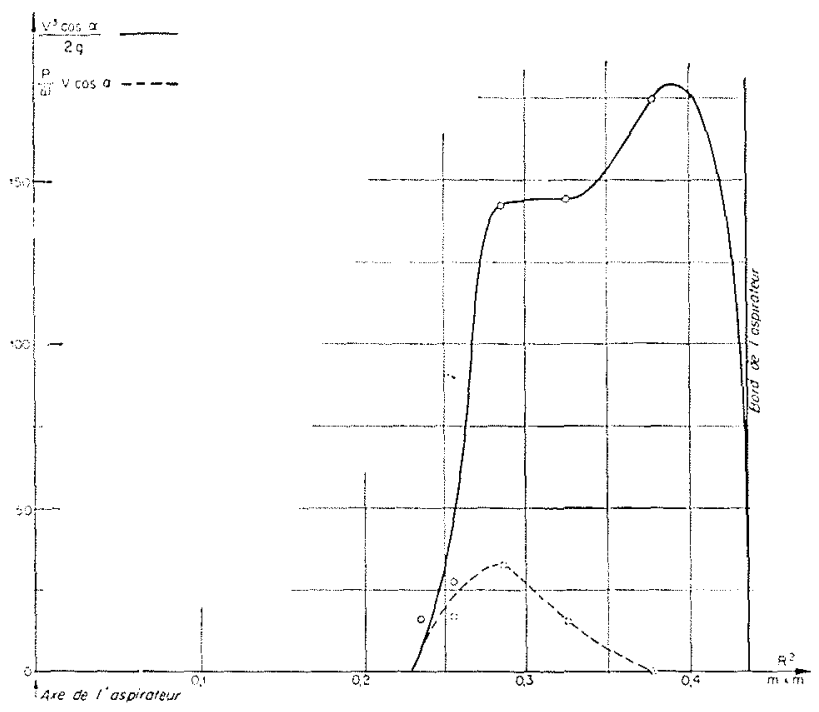

Fic. $4 a$. Bromate (groupe 4).

Valeurs de $\frac{V^{\prime \prime} \cos \alpha}{2 g}$ et $\frac{P}{\bar{\omega}} V \cos \alpha$ a $10.000 \mathrm{~kW}$ (V en $\mathrm{m} / \mathrm{sec}, \frac{p}{\dddot{\omega}}$ en mètres d'eau).

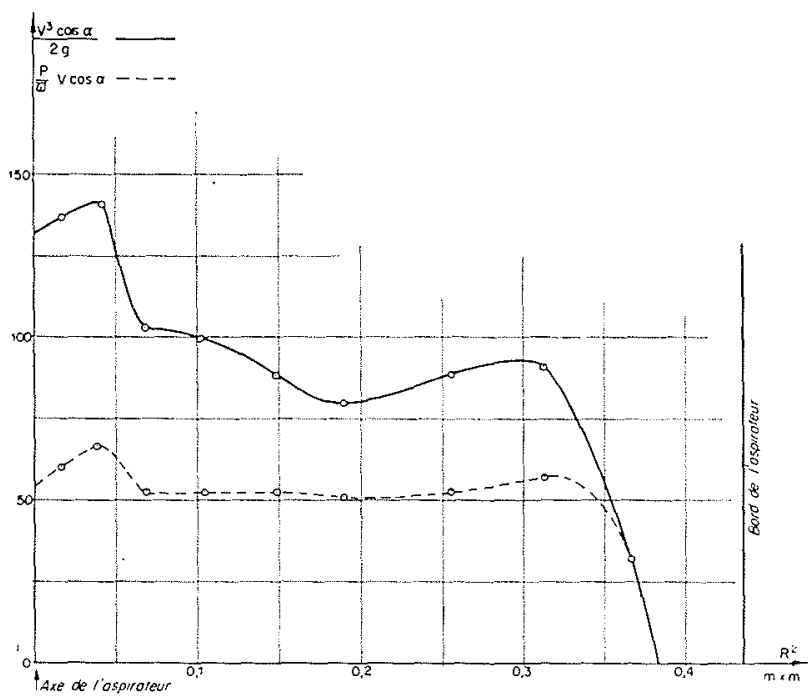

Fici. 4 c. - Bromiat (groupe 4).

Valeurs de $\frac{V^{3} \cos \alpha}{2 g}$ et $\frac{\mathrm{P}}{\bar{\omega}} \mathrm{V} \cos \alpha$ it $25.000 \mathrm{~kW}$

$\left(V\right.$ en $\mathrm{m} / \mathrm{sec}, \frac{\mathrm{P}}{\bar{\omega}}$ en mètres d'calu.

\section{5" Remarques sur le fonctionnement DE L'ASPIRATEUR}

En dehors des considérations énergétiques, nous avons eu l'occasion de faire les quelques remarques suivantes :

a) A la sortie de la rone, l'eau possède une vitesse tangentielle qui, pour certaines charges, est loin d'être négligeable (composantes tangen-

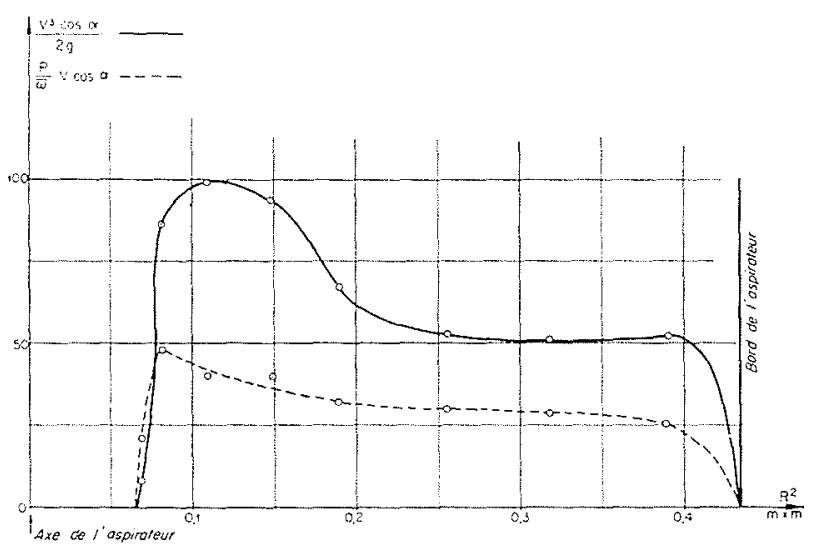

Fig. $4 b$. - Bnommat (groupe 4 ). Valeurs de $\frac{V^{3} \cos \alpha}{2 g}$ et $\frac{P}{\bar{\omega}} V \cos \alpha a 20.000 \mathrm{~kW}$ $\left(V\right.$ en $\mathrm{m} / \mathrm{sec}, \frac{\mathrm{P}}{\bar{\omega}}$ en mètres d'eau).

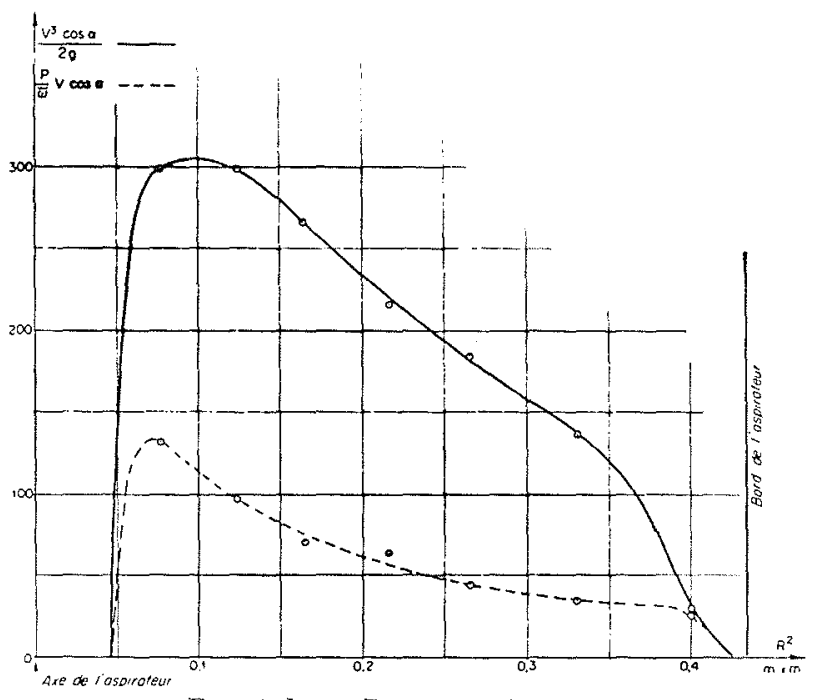

Fig. 4 d. - Brommat (groupe 4).

Valeurs de $\frac{V^{3} \cos \alpha}{2 g}$ et $\frac{P}{\bar{\omega}} V \cos \alpha$ a $30.000 \mathrm{~kW}$ ( $V$ en $\mathrm{m} / \mathrm{sec}, \frac{\mathrm{P}}{\bar{\omega}}$ ch mètres d'eau). 
tielles et verticales sensiblement égales pour $10.000 \mathrm{~kW}$ ). L'eau aborde donc le coude de l'aspirateur avec un mouvement qui peut s'apparenter à un mouvement hélicoïdal, le sens de rotation variant suivant la charge (sens de rotation de la roue pour les faibles charges, sens inverse pour les fortes charges).

Si l'on suit alors les trajectoires des divers filets liquides régulièrement répartis à l'entrée du cône d'aspiration, en admettant la conservation du pas de l'hélice pour chaque filet, on constate à la sortie de l'aspirateur une accumulation des trajectoires dans une zone déterminée, variable avec le pas de l'hélice (donc avec la puissance développée par la turbine). Cette accumulation est provoquée par la dissymétrie importante introduite par le coude entre les diverses trajectoires.

L'hypothèse de base n'est évidemment qu'approchèe, mais cette remarque suffit peul-être à expliquer un phénomène que l'on peut fréquemment observer : dans de nombreuses turbines Francis à axe vertical, dont la sortie de l'aspirateur est divisée en deux pertuis, on constate que :

- Pour les faibles charges, le débit se concentre dans un seul des pertuis;

- Pour les fortes charges, e'est le deuxième pertuis qui est presque exclusivement inléressé;

- Pour une charge intermédiaire bien délerminée, l'eau se répartit à peu près uniformément entre les deux pertuis.

b) Au cours des mesures, on remarque à cerlaines charges :

- Des diminutions passagères de la pression dans le cône d'aspiration;

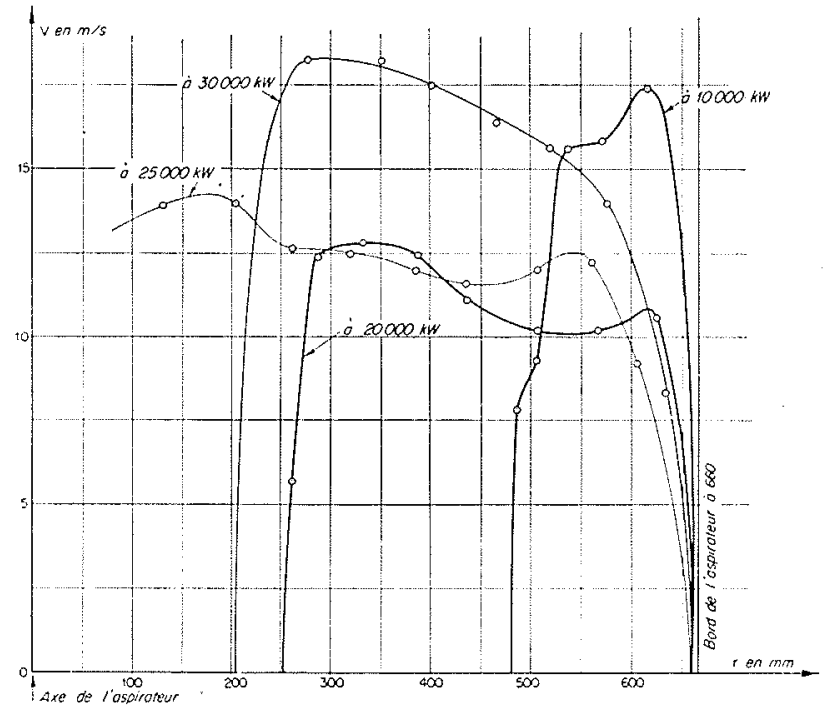

Fici, t bis. - Bromat (groupe 4).

Repartition des vilesses sur un rayon de l'aspirateur pour différentes charges.

- Des changements passagers dans le bruit de l'aspiration;

- Des baisses passagères de la température de l'eau soutirée dans le canal de fuite.

Ces observations pourraient s'interpréter par des désamorcages de l'aspirateur plus ou moins prononcés, suivis de réamorçages instables et de plus courte durée, ces derniers se traduisant par une augmentation de la dépression et une diminution des pertes (d'où augmentation de rendement et diminution de la température de l'eau).

Cette hypothèse expliquerait également les sortes de "bouffees » d'cau que l'on peut observer à la sortie de certaines turbines.

\section{IV. - PERTES DANS LE ROBINET SPHERIQUE, LA BACHE, LE DISTRIBUTEUR ET LA ROUE}

\section{1" Mesure indrecte des pertes}

On peut obtenir ces pertes par différence entre les pertes totales et les pertes partielles précédemment étudiées.

Les pertes tolales ont été déterminées par la méthode thermométrique :

$$
\varepsilon=100\left(\frac{1-427\left(\theta_{4}^{\prime}-0.2\right)}{\mathrm{H}_{n}}\right)
$$

$\approx$ pertes totales exprimées en \% de l'énergie hydraulique totale. $0_{2}^{\prime}$ température de l'eau soutirée en amont de la turbine el ramenée à la pression atmosphérique, à la cote de restitution et à une vitesse négligeable.

$\theta_{2}$ température de l'eau soutirée en aval de la turbine et ramence aux mèmes conditions que ci-dessus. Le soutirage est effectué avec un tube de PITot placé à la sorlie de l'aspirateur de maniere à récupérer l'énergie cinétique résiduelle (énergie qui est done comprise dans cette mesure des pertes totales).

$\mathrm{H}_{n}$ hauteur de chute netle. 
Les mesures de $0_{2}$ ont été faites en plusieurs points du canal de fuite (situés sur une verticale).

D'autre part, notons que, au cours des mesures, l'eau de fuite du labyrinthe supérieur était entièrement refoulée dans les circuits de réfrigération. Par suite, il faut ajouter les pertes correspondantes aux résultats obtenus dont une partie est, à titre d'exemple, consignée dans les tableaux ci-dessous (groupe $n^{\circ} 4$ ) :

Puissance aux bonnes de l'alternateun : $30.000 \mathrm{~kW}$

\begin{tabular}{|c|c|c|c|c|}
\hline $\begin{array}{c}\mathrm{H}_{n} \\
(\mathrm{en} \mathrm{m})\end{array}$ & $\theta_{2}^{\prime}$ & $\theta_{2}$ & $\theta$ & $(\%)$ \\
\hline 239 & $\begin{array}{l}4,075 \\
4,075 \\
4,065 \\
4,065\end{array}$ & $\begin{array}{l}3,57 \\
3,565\end{array}$ & $\underbrace{0,50 \overline{3}}_{0,50} 0,502$ & 10,3 \\
\hline 239 & $\begin{array}{l}4,06 \\
4,06 \\
4,05 \\
4,05\end{array}$ & $\begin{array}{l}3,555 \\
3,54\end{array}$ & $0,0,505)$ & 9,2 \\
\hline 239 & $\begin{array}{l}4,05 \\
4,055 \\
4,05\end{array}$ & $\begin{array}{l}3,555 \\
3,55\end{array}$ & 0,503 & 10,1 \\
\hline 237 & $\begin{array}{l}4,06 \\
4,055 \\
4,06\end{array}$ & $\begin{array}{l}3,55 \\
3,55\end{array}$ & 0,507 & 8,7 \\
\hline 238 & $\begin{array}{l}4,05 \\
4,055 \\
4,05\end{array}$ & $\begin{array}{l}3,555 \\
3,555\end{array}$ & 0,497 & 10,8 \\
\hline 237 & $\begin{array}{l}4,05 \\
4,05 \\
4,05\end{array}$ & $\begin{array}{l}3,55 \\
3,545\end{array}$ & 0,503 & 9,4 \\
\hline \multicolumn{4}{|c|}{ Moyenne........ } & 9,7 \\
\hline
\end{tabular}

On peut ainsi tracer les courbes (fig. 5) qui résument tous les résultats obtenus :

Notons que la mesure des pertes totales à $10.000 \mathrm{~kW}$ est sujette à caution, les divers résultats présentant un écart atteignant $4 \%$ sur le

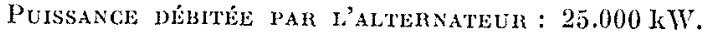

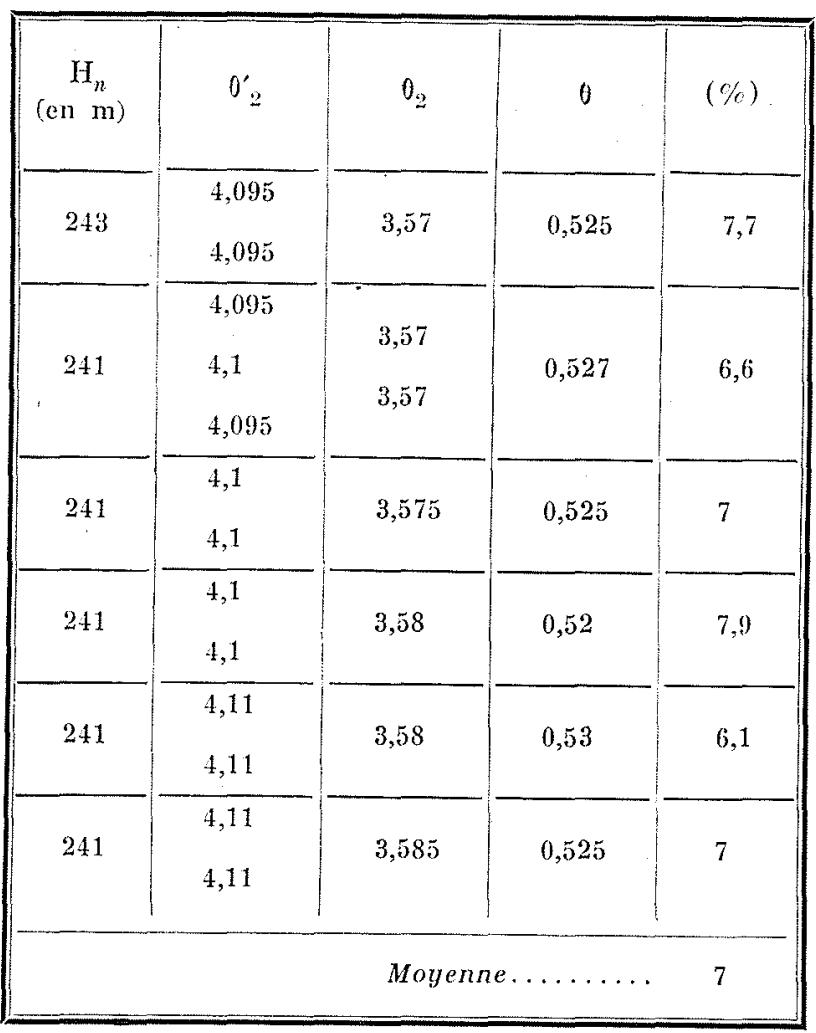

Púrssance débitée par L'altennateur : $20.000 \mathrm{~kW}$.

\begin{tabular}{|c|c|c|c|c|}
\hline $\begin{array}{c}\mathrm{H}_{n} \\
(\mathrm{en} \mathrm{m})\end{array}$ & $\theta_{2}^{\prime}$ & $0_{2}$ & $\theta$ & $(\%)$ \\
\hline 242 & $\begin{array}{l}4,12 \\
4,12\end{array}$ & 3,6 & 0,52 & 8,2 \\
\hline 242 & $\begin{array}{l}4,13 \\
4,13\end{array}$ & 3,61 & 0,52 & 8,2 \\
\hline 242 & $\begin{array}{l}4,13 \\
4,13 \\
4,13\end{array}$ & $\begin{array}{l}3,615 \\
3,62\end{array}$ & 0,513 & 9,5 \\
\hline 242 & $\begin{array}{l}4,135 \\
4,135\end{array}$ & 3,62 & $0, \overline{\mathrm{i}} 15$ & 9,1 \\
\hline 242 & $\begin{array}{l}4,135 \\
4,135\end{array}$ & 3,62 & 0,515 & 9,1 \\
\hline \multicolumn{4}{|c|}{ Moyenne....... } & 8,8 \\
\hline
\end{tabular}

rendement. Des considérations d'exploitation ne nous permettant pas de reprendre ces mesures dans un proche avenir, nous préférons donner un résultat incertain et ne pas retarder la publication de ce compte rendu. 


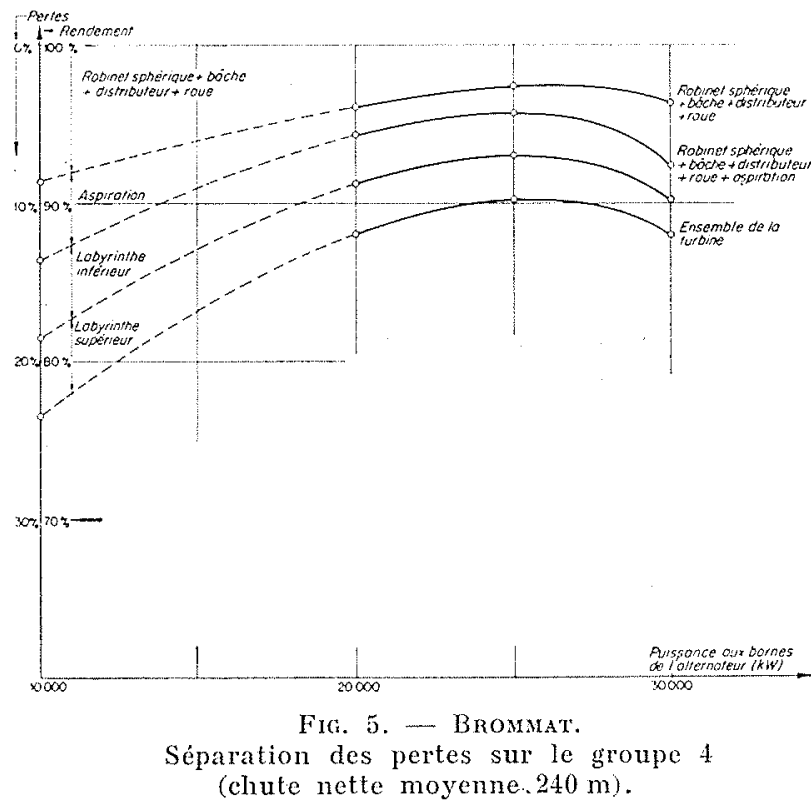

A titre indicatif, nous avons recherché le renrement de la roue du groupe $n^{\circ} 6$ :

--- A partir d'une courbe de rendement établie aux moulinets (pour une hateur de chute nette moyenne de $225 \mathrm{~m}$ );

-- En négligeant les pertes par frottement dans le palier turbine (de lordre de $10 \mathrm{~kW}$, donc négligeables);

-. En admettant que les pertes dans l'aspirateur sont identiques à celles mesurées sur le groupe 4 (mesures faites avec une hauteur de chute nette moyenne de $240 \mathrm{~m}$ et une aspiration moyenne de $1 \mathrm{~m}$ ).

Les résultats obtenus sont consignés sur les courbes $n^{\circ} 6$.

Ces courbes mettent en évidence un résultat important : le rendement de l'ensemble, robinet sphérique + bâche + distributeur + roue, atteint des valeurs élevées (de l'ordre de $97 \%$ ) et ce rendement ne décroît que lentement quand la charge diminue.

On peut donc concevoir des turbines Francis susceptibles de fonctionner sous charge réduite avec des rendement tout a fait acceptables si l'on arrive à réduire l'importance des pertes dans l'aspiration (ce qui est probable) et surtout des pertes dans les labyrinthes.

L'importance des « labyrinthes réduits 》 se trouve ainsi remise en évidence, plus particulièrement pour les usines comportant des groupes de faible vitesse spécificue et devant fonctionner a des charges tries variables.

Remarour. - Toutes les mesures de pertes ont cité faites :

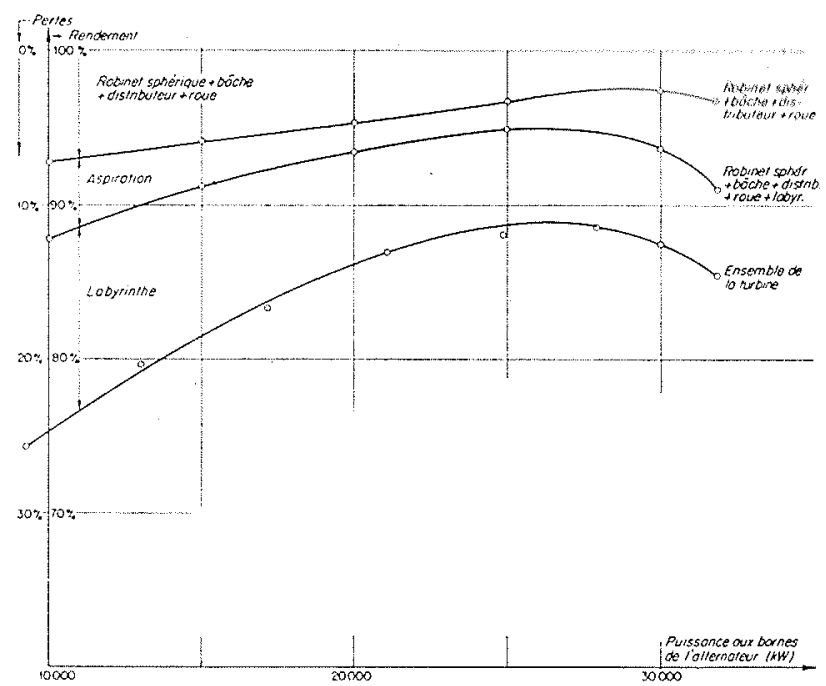

Fig. 6. - Brommat.

Séparation des pertes sur le groupe 6 .

- Sous des hauteurs de chute nette voisines de $240 \mathrm{~m}$;

- Arec des hauteurs d'aspiration voisines de $1 \mathrm{~m}$.

(Hauteur d'aspiration : différence entre la cote moyenne de la turbine et la cote de restitution, différence comptée positivement si le plan d'eau aval est au-dessous de la turbine.)

Nous navons pas tenu compte des variations des hauteurs de chute et d'aspiration autour de ces valeurs moyennes, car, à Brommat, leur importance relative est faible.

Il serait cependant très intéressant d'étudier la variation des diverses catégories de pertes en fonction de ces paramètres. Cette étude serait particulièrement indiquée pour les turbines basses et moyennes chutes.

Notons enfin que les mesures des hauteurs de chute et des puissances ont été faites d'après les appareils indicateurs de l'usine.

\section{$2^{\circ}$ Mesure DIRECTE DES PERTES}

On peut mesurer directement les pertes dans l'ensemble rohinet sphérique + bâche - distributeur + rone en utilisant la méthode thermométrique.

\section{Appelons :}

$\theta_{1}$ ìa température de l'eau à l'entrée de la turbine (température supposée constante dans la section de mesure; sinon $0_{1}$ désigne une valeur moyenne obtenue par exemple par intégration graphique). 
o la température de l'eau en un point $M$ de ( () (rappelons que $(\Sigma)$ désigne une section de l'aspirateur immédiatement à la sortie de la roue).

$d Q$ le débit traversant un élément $d \sigma$ de ( $)$ entourant le point $M$.

dq la puissance évacuée sous forme calorifique par le débit $d Q$.

On a :

$$
d q=\left(0-0_{1}\right) \cdot d Q=\left(0-0_{1}\right) v \cos \alpha d \sigma
$$

D'ò̀ :

$$
q==\iint_{(\Sigma)}\left(\theta-\theta_{1}\right) v \cos \alpha d \sigma
$$

La puissance totale $q$ correspond sensiblement aux pertes dans le robinet sphérique, la bâche, le distributeur et la roue.

Malheureusement, la mesure directe de 0 et $D_{1}$ présente certaines difficultés.

On sait ${ }^{1}$ que l'on peut calculer $\theta_{1}$ en soutirant de l'eau à l'entrée de la turbine et en me- surant la température $\theta_{2}^{\prime}$ de cette eau détendue dans un récipient approprié.

Si le récipient est placé à la cote de restitution et si la vitesse de l'eau à la sortie du récipient est négligeable, on a :

$$
\theta_{1}=\theta_{2}^{\prime}-\frac{\mathrm{H}_{n}}{427}
$$

$\left(\mathrm{H}_{n}\right.$ hauteur de chute nette).

Par analogie, soutirons de l'eau en $M$ avec un tube de Pitor, détendons la comme ci-dessus à la cote de restitution et mesurons sa température O.p. Toute l'énergie que l'eau possédait à l'entréc du tube de Pitor s'est transformée en chaleur. Cette énergie ayant été admise égale à :

$$
\operatorname{dm}\left(\frac{v^{2}}{2 g}+\frac{p}{\varpi}+z\right)
$$

On a :

$$
0=0.21,-\frac{1}{427}\left(\frac{v^{2}}{2 g}+\frac{p}{\sigma 1}+z\right)
$$

$$
\text { D'où : } \quad q=\iint_{(\Xi)}\left(\theta_{2 \mathrm{P}}-\theta_{1}\right) v \cos \alpha d \sigma-\frac{1}{427} \iint_{(\mathrm{z})}\left(\frac{v^{2}}{2 g}+\frac{p}{\bar{\omega}}+z\right) v \cos \alpha d \sigma
$$

Nous remarquons que le deuxième terme correspond aux pertes dans l'aspirateur.

D'autre part, le premier terme représente la puissance totale évacuée sous forme calorifique par l'eau traversant la turbine quand on dissipe dans un tube de Prró toute l'énergie résiduelle de l'eau à la sortie de la roue.

Or, cette mème dissipation d'énergie est également réalisée dans l'aspirateur et le tube do Prót utilisé pour le soutirage dans le canal de fuite. On a :

$$
\iint_{(2)}\left(0_{2 \mathrm{P}}-0_{1}\right) v \cos \alpha d \sigma=\mathrm{Q}\left(0_{2}-0_{1}\right) \text { - pertes aux labyrinthes (éventuellement). }
$$

On est donc ramené à l'évaluation des pertes par différence.

L'intérêt de ces mesures est l'étude de la répartition des températures 0 à l'entrée de l'aspirateur.

A titre d'exemple, nous donnons cette répartition pour la puissance maximum 0, répartition caractérisée par :

$$
\theta_{2}^{\prime}-\theta=\frac{H_{n}}{427}-\left(\theta-\theta_{1}\right)
$$

On peut observer trois zones différentes : A, $B$ et $C$, figure 7 .

- En A, l'eau « chaude » en provenance du labyrinthe inférieur se mélange progressivement à l'eau qui a traverse la roue et

1. La Houlle Blanche, janvier-février 1951 : « Quelques applications de la méthode thermométrique POIRSON $\gg$.

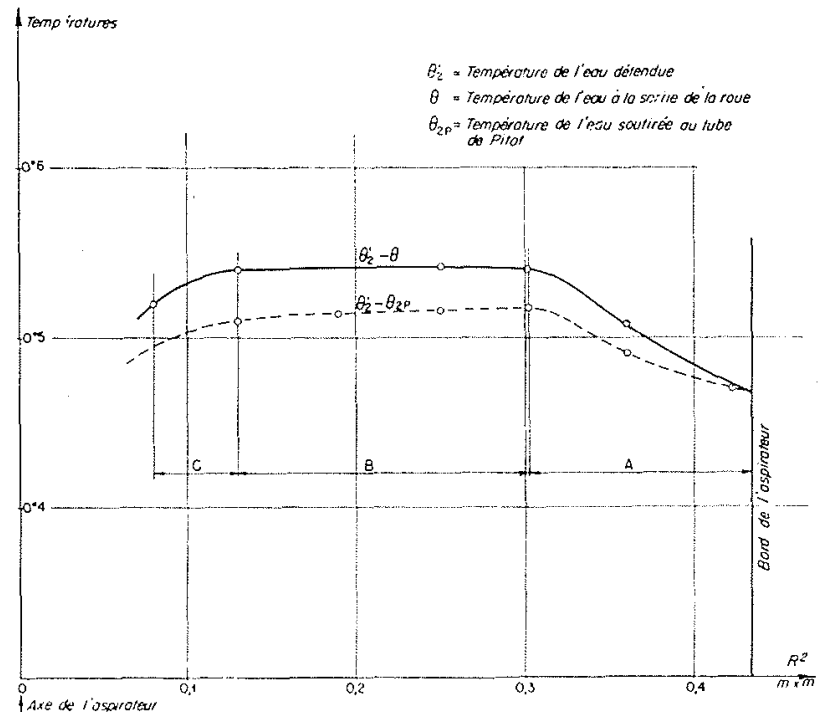

Fig. 7. - Brommat (groupe $4.30 .000 \mathrm{~kW}$ ). Répartition des températures à l'entrée de l'aspirateur. 
provoque les élévations de température observées.

- En B, on note une température pratiquement constante, probablement indépendante des pertes dians le labyrinthe inféricur.

- Enfin C correspond au contact entre une «zone d'écoulement» et une «zone relativement morte $»$. Ce contact s'accompagne probablement de tourbillons, d'où dissipation d'énergie et élévation de température.

Remarque. - La mesure des températures 0 peut également constituer un contrôle des résultats obtenus par la mesure indirecte des pertes: méthodes en réalité analogues mais mesures différentes.

A titre de vérification, nous avons calculé le rendement de l'ensemble robinet sphérique + bâche + distributeur + roue, a partir des valeurs de $\theta$ mesurées dans la zone $B$ (en admettant que la température de l'eau serait uniforme et égale à sa valeur dans la zone $\mathbf{B}$, si on supprimait l'influence des pertes dans le labyrinthe inférieur et l'aspiration).

Les résultats, mesurés dans le tableau ci-dessous, donnent une concordance satisfaisante pour les moyens de mesure employés.

\section{GROUPE N4}

\begin{tabular}{|c|c|}
\hline $\begin{array}{c}\text { Puissance aux bornes } \\
\text { de l'alternateur }\end{array}$ & $\begin{array}{c}\text { Rendement roue + distributeur } \\
+ \text { bâche + robinet sphérique }\end{array}$ \\
\hline $30.000 \mathrm{~kW}$ & $96 \%$ \\
$25.000 \mathrm{~kW}$ & $97,8 \%$ \\
$20.000 \mathrm{~kW}$ & $96,8 \%$ \\
\hline
\end{tabular}

Notons que, théoriquement, on devrait pouvoir vérifier que l'accroissement de température observé dans la zone A correspond bien aux pertes du labyrinthe inférieur. Pratiquement, la précision des appareils de mesure utilisés ne permet pas cette vérification.

Enfin, pour les faibles charges, la largeur de la zone d'écoulement diminue rapidement et la région $B$ disparaît. Aussi ne peut-on plus séparer comme ci-dessus les pertes de la rone de celles du labyrinthe inférieur. En évaluant les pertes dans toute la section d'écoulement et en retranchant du résultat les pertes en provenance du labyrinthe inférieur, on trouve :

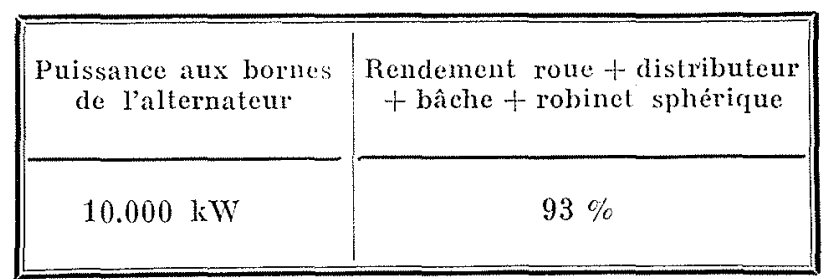

Ce qui confirme le bon rendement de la roue a faible charge.

\section{CONCLUSION}

L'étude sommaire que nous avons faite demande à ètre perfectionnée :

- Par des moyens d'investigation plus poussés, en particulier par l'utilisation d'appareils de mesure plus précis (nous savons parfaitement que les appareils en notre possession sont parfois nettement insuffisants);

- Par l'étude de l'influence des facteurs autres que la puissance débitée : hauteur de chute, hauteur d'aspiration par exemple;

- Par des mesures sur des turbines différentes.

Notons en particulier qu'à l'occasion des essais de réception de Couesque, on pourrait effectuer des mesures pleines d'intérèt, montrant en particulier la répartition des pertes sur des turbines de même puissance qu'à Brommat, mais de vitesse spécifique différente ${ }^{1}$.

Cependant, nous croyons dès à présent avoir mis en évidence la forme du spectre des vitesses et l'importance des angles de sortie sous la roue, ainsi que la valeur relative élevée des pertes dans les labyrinthes et l'aspiration. Les gains de rendement que l'on peut espérer réaliser en diminuant ces pertes semblent justifier des études plus approfondies de ces phénomènes.

D'autre part, les principes de cette séparation des pertes sur les turbines Fravcrs peuvent être transposés pour étudier d'autres types de turbines.

La méthode peut s'adapter très facilement à l'étude des turbines KAPLAN si l'on peut faire des mesures de températures suffisamment précises (avec des thermo-couples ou, de préférence, des thermistors).

Enfin, dès à présent, on peut envisager de séparer sur les turbines PELton :

- Les pertes dans la buse,

- Les pertes dans la roue,

- L'énergie hydraulique résiduelle.

1. Vitesse spécifique :

à Brommat . . . 100

à Couesque. . . . 300 


\section{ANNEXE}

Depuis l'établissement de notre première note, nous avons poursuivi nos essais et notamment à l'usine de Couesque, en collaboration avec MM. Bonner el CampMas, sur une turbine de $42.000 \mathrm{ch}$ $-\mathrm{N}_{\mathrm{s}}=300$ sous $60 \mathrm{~m}$ de chute.

Dans ces essais, les pertes dans laspiration ont été caractérisées par $\frac{h}{\mathrm{H}}$ avec $\mathrm{H}=$ chute brute

$$
h=Z+\frac{\int_{0}^{\mathrm{R}} \frac{v^{3} \cos \alpha}{2 g} d r^{2}}{\int_{0}^{\mathrm{R}} v \cos \alpha d r^{2}}+\frac{\int_{0}^{\mathrm{R}} \frac{\mathrm{P}}{\mathrm{\omega}} v \cos \alpha d r^{2}}{\int_{0}^{\mathrm{R}} v \cos \alpha d r^{2}}
$$

Les courbes obtenues après dépouillement rapide des résultats et en particulier la courbe $D$ montrent que les fuites dans l'aspiration varient très rapidement et croissent d'une maniere importante au-delà de la charge optima.

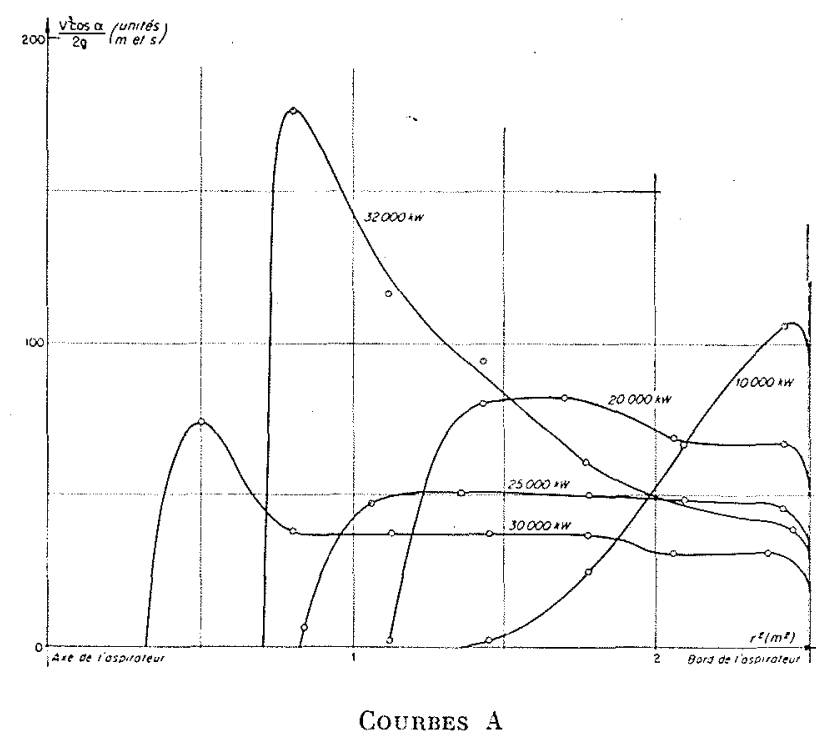

Répartition de l'énergic cinétique de l'eau sur un rayon de l'aspirateur pour diverses puissances débitées par lalternateur (Couesque : groupe $n^{\circ} 2$ ).

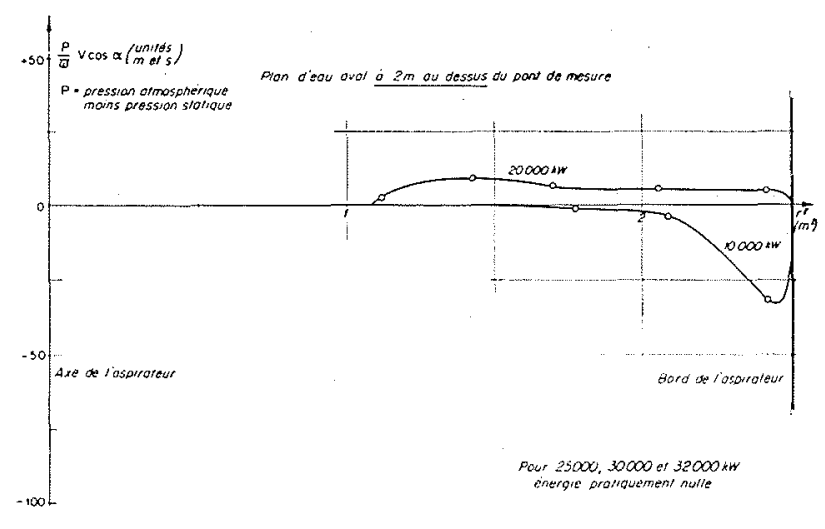

Courbes B

Répartition de l'énergie de pression sur un rayon de l'aspirateur pour diverses puissances débitées par l'alternateur (Couesque : groupe $\mathrm{n}^{\circ} 2$ ).

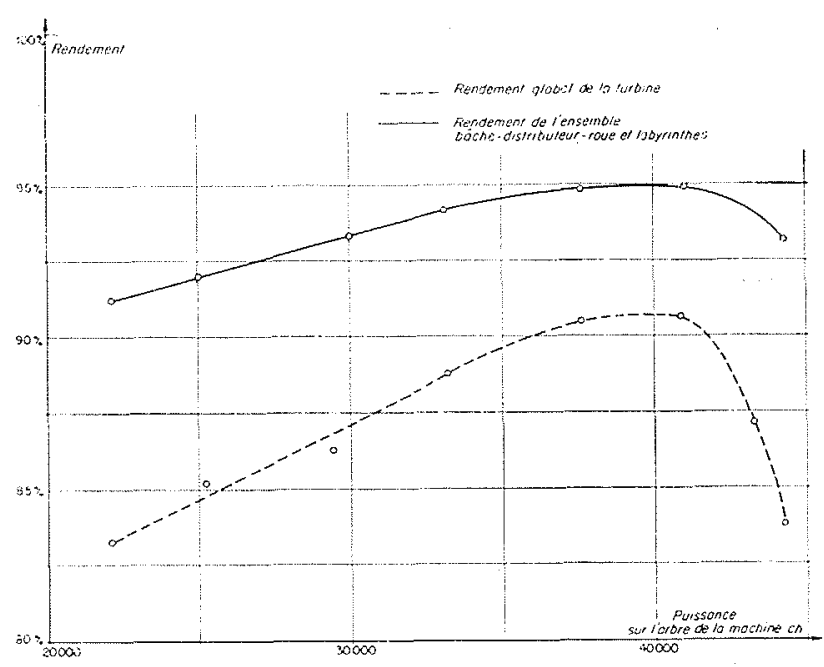

Covmues $\mathrm{C}$

(Couesque: groupe $\mathrm{n}^{0} 2$ ).

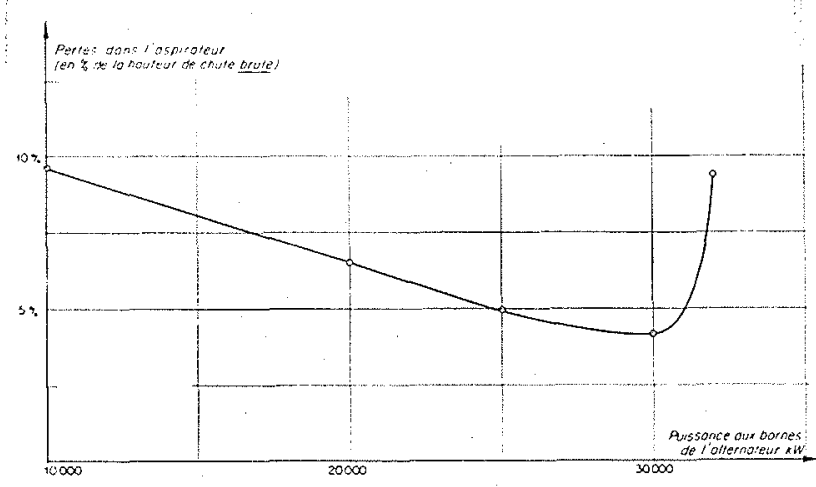

Courbes D

Pertes de l'aspirateur en fonction de la puissance débited (Couesque : groupe $n^{\circ} 2$ ). 